\title{
On-Line Scanned Probe Microscopy Transparently Integrated With Twin SEM/FIB Systems
}

\author{
Aaron Lewis ${ }^{1}$, Andrey Ignatov ${ }^{2}$, Anatoly Komissar ${ }^{2}$, Hesham Taha $^{2}$ and Eran Maayan ${ }^{2}$ \\ ${ }^{1}$ Hebrew University of Jerusalem, Jerusalem, Israel \\ ${ }^{2}$ Nanonics Imaging Ltd, Jerusalem, Israel
}

Scanning electron microscopy (SEM) is a moving force in the nanotechnological revolution. Focused ion beam microscopes (FIB) have also become potent in nanotechnology and their combination with SEMs have shown the power of such on-line combinations. We describe in this paper the transparent integration in SEM/FIB of another enabling imaging \& sensing technology, scanning probe microscopy (SPM). This is accomplished without affecting any detectors, injectors, analyzers or obscuring the sample stage of such twin beam systems. The transparent combination is accomplished so that the probe does not obscure the election/ion beam axis and also sits at the eucentric point. This permits the SPM to rotate into position when either the electron or ion beam is in place for standard normal operation relative to the sample surface. Such a Triple Beam ${ }^{\mathrm{TM}}$ combination is a disruptive technology affecting the potential of both electron, ion and scanned probe applications. It will be shown that it is now possible to rapidly place an SPM probe at a nanometric position within a large field of view to provide for ultrahigh resolution protocols unavailable in a SEM or FIB such as nanometric $\mathrm{Z}$ imaging or regions of charging in a sample. The combination effectively allows for a variety of 3D functional SPM imaging possibilities with on-line FIB material slicing. This is accomplished while allowing for deep trench profiling and side wall imaging enabled by unique SPM and probe design. Such new directions in functional understanding of materials will be discussed in this presentation while monitoring probe tip characteristics and often effectively repairing the probe tip on-line. The lack of these possibilities has limited SPM technology. A NanoTool Kit ${ }^{\mathrm{TM}}$ of electron/ion beam friendly probes with a wide spectrum of functionality will be described based on singular glass probe technology. Examples will cover on-line measurements of elasticity, electrical, thermal and even super-resolution optical imaging of cathodoluminescence and biomaterial staining. The integration described in this talk portends new directions of application in fundamental and applied science not previously accessible. 